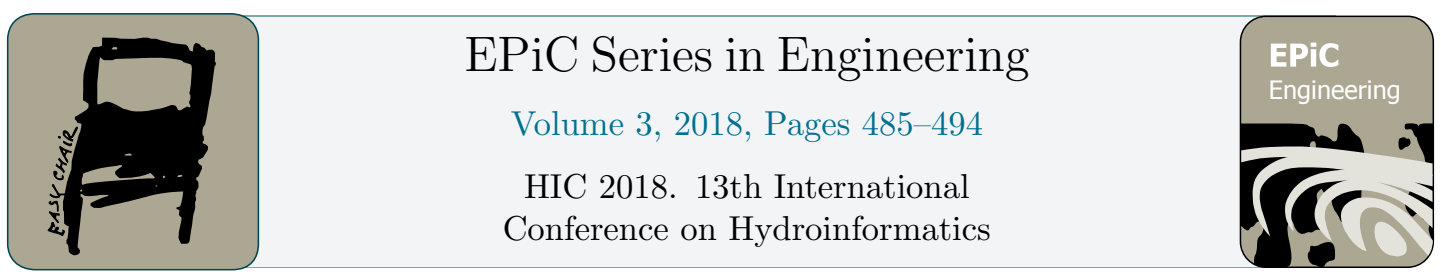

\title{
Resilience analysis in the permanent partitioning of a water distribution network
}

\author{
E. Creaco $^{1,3}$, A. Di Nardo ${ }^{2,3,4}$, C. Giudicianni ${ }^{2}$, R. Greco ${ }^{2,3}$, G.F. Santonastaso ${ }^{2,3}$ \\ ${ }^{1}$ Università di Pavia, Pavia, 27100, Italy \\ ${ }^{2}$ Università della Campania Luigi Vanvitelli via Roma n. 29, Aversa, 81031, Italy, \\ ${ }^{3}$ Action Group CTRL+SWAN of the European Innovation Partnership on Water, \\ via Roma n. 29, Aversa, Italy \\ ${ }^{4}$ Istituto Sistemi Complessi (CNR), via dei Taurini 19, Roma, 00185, Italy
}

\section{Corresponding author: armando.dinardo@unicampania.it}

\begin{abstract}
This paper aims to explore the suitability of compact resilience metrics for application to partitioned water distribution networks (WDNs). WDN partitioning represents a different test from the usual reliability tests performed in the scientific literature, in which the operation of the WDN is unperturbed, or marginally perturbed (e.g., by segment isolation or demand amplification). The creation of permanent district metering areas (DMAs), which is carried out through the simultaneous closure of numerous links, represents, instead, a larger and permanent perturbation that deserves special attention. In this analysis, two metrics, namely the Global Resilience Failure (GRF) and the energy efficiency indices, were compared in pressure-driven approach with WDN performance indicators. The results in a real WDN, which is partitioned in a growing number of DMAs, proved that both the GRF is more sensitive to the weaknesses arisen in the partitioning processes.
\end{abstract}

Keywords: failure, DMA, redundancy, resilience, water network partitioning

\section{Introduction}

In the last years, many studies have attempted to define mathematically the reliability of water distribution networks, as their ability to keep suitable standards of service to users in normal and critical operation scenarios.

As surrogates for reliability, the concepts of resilience and redundancy have been presented. The former is related to the energy storage that the network has under normal operating conditions, which can be used as a backup to tackle critical scenarios, such as those related to component failures [1]. The latter, instead, is more related to 
the number of paths that connect the network source(s) to the generic demanding node; in fact, the abundance of paths may be a precious resource for the network to tackle critical scenarios [2].

In relation to water distribution networks, resilience is linked to the network geometry (shape and dimension) and topology (node degree, loops, etc.), and to hydraulic characteristic of network components (diameter, roughness, length, etc.). Because of these multi-dependent factors, there is no single established approach or method to assign and to quantify resilience.

Recently, some metrics have been proposed in the literature based on energy and topological approaches $[3,4,5,6,7]$. Anyway, these metrics are not able to analyse exhaustively all aspects of the complex behaviour of water distribution networks. Furthermore, most works studied the effectiveness of reliability surrogate metrics on networks perturbed by demand variations and random pipe failures [8]. Evidently, these perturbations can be described as critical operational scenarios that occur occasionally. The issue of how these metrics behave in the case of network partitioning (that is the creation of permanent District Meter Areas DMAs), instead, has not been thoroughly investigated. As is known, the division of a Water Distribution Network (WDN) into $N_{d}$ DMAs or subsystems (clusters) simplifies and improves the following management aspects: water loss reduction, pressure management, active leakage control, maintenance of pipes and water quality, in terms of protection from intentional or accidental contaminations [9].

To take these advantages, it is necessary divide physically the network by closing many pipes and inserting flow meters in feeding pipes of DMAs. However, permanent DMAs worsen the hydraulic performance of water network and change significantly the original topological layout. Therefore, the water network partitioning represents a complex challenge for engineers and Water Utilities. To this purpose, a definition of resilience measure able to evaluate the effects of permanent DMAs coupled with economic considerations [10] could be very useful in a decision support system to compare easily different solutions of partitioning. Therefore, the responsiveness of resilience metrics, available in the literature, to detect the perturbations imposed by permanent DMAs deserves special attention in this paper.

\section{Method}

The metrics compared in this paper are based on considerations on power and energy [11]. to assess the reliability of water distribution system and employ the total inlet power or energy supplied by water sources or pumping station. To measure WDN resilience, the WDN behaviour was carried out in PDA (pressure driven analysis) [12]. To this end, the most adequate resilience metrics found in the literature are:

a) Energy efficiency index proposed in [13]: 
$e=\frac{\mathbf{q}_{\text {user }}^{\mathrm{T}} \mathbf{H}}{\mathbf{Q}_{0}^{\mathrm{T}} \mathbf{H}_{\mathbf{0}}+\mathbf{Q}_{\mathbf{p}}^{\mathrm{T}} \mathbf{H}_{\mathbf{p}}}$

b) Global Resilience Failure index (GRF) proposed in [12]:

$$
G R F=I_{r}+I_{f}
$$

with

$$
I_{r}=\frac{\max \left(\mathbf{q}_{\text {user }}^{\mathbf{T}} \mathbf{H}-\mathbf{d}^{\mathrm{T}} \mathbf{H}_{\mathrm{des}}, 0\right)}{\mathbf{Q}_{\mathbf{0}}^{\mathrm{T}} \mathbf{H}_{\mathbf{0}}+\mathbf{Q}_{\mathbf{p}}^{\mathrm{T}} \mathbf{H}_{\mathbf{p}}-\mathbf{d}^{\mathbf{T}} \mathbf{H}_{\mathrm{des}}}
$$

and

$I_{f}=\frac{\min \left(\mathbf{q}_{\text {user }}^{\mathrm{T}} \mathbf{H}-\mathbf{d}^{\mathrm{T}} \mathbf{H}_{\mathrm{des}}, 0\right)}{\mathbf{d}^{\mathrm{T}} \mathbf{H}_{\text {des }}}$

where $\mathbf{q}_{\text {user }}$ and $\mathbf{d}$ are the vectors of the water discharges delivered to the users and the users' demands, respectively. $\mathbf{H}, \mathbf{H}_{\mathbf{0}}$ and $\mathbf{H p}$ are the vectors of total heads at demanding nodes, water sources and pumps, respectively. $\mathbf{Q}_{\mathbf{0}}$ and $\mathbf{Q}_{\mathbf{p}}$ are the vectors of supplied water discharges by water sources and pumps, respectively. Finally, $\mathbf{H}_{\text {des }}=\mathbf{z}+\mathbf{h}_{\text {des, }}$, where $\mathbf{z}$ and $\mathbf{h}_{\text {des }}$ are the vectors of nodal ground elevations and nodal $h_{\text {des }}$, respectively.

The energy efficiency index (Eq. 1), represents the ratio of power supplied to the users to the total power delivered by water sources and pumping stations. The $G R F$ (Eq. 2) combines two useful indices: resilience index and failure index. The former is the ratio of the hydraulic power surplus that could be dissipated in case of failures to the maximum power surplus available in a WDN; the latter is a measure of power deficit computed as the ratio of the hydraulic power deficit to the minimum required power at demand node.

The comparison of the two resilience metrics was carried out using topological and hydraulic indices in order to understand the capacity of each resilience metric to catch or highlight peculiar behaviour of the partitioned systems. To this aim, the loop diameter uniformity coefficient $C_{u}$ [7] and the demand satisfaction rate $I_{s}$ [14] are computed. The topological index $C_{u}$, is an index of uniformity of pipes in WDN loops, computed as follows: 


$$
C_{u}=\frac{n_{\text {pwithloop }}}{n_{p}} \frac{\sum_{l=1}^{n_{l}} C_{l}}{n_{l}}
$$

where, $n_{\text {pwithloop }}$ is the number of pipes that belongs at least at one loop (pipes forming branch structure are not take account in $n_{\text {pwithloop }}$ ), $n_{p}$ is the total number of pipes in network, $C_{l}$ is the ratio between the mean and maximum diameter of generic loop. The hydraulic index $I_{s}$, which is an indicator of the WDN performance, can be defined as follows:

$$
I_{s}=\frac{\sum_{i=1}^{n} q_{i}}{\sum_{i=1}^{n} d_{i}} \cdot 100
$$

where, $q_{i}$ and $d_{i}$ are respectively the water supplied to users, calculated on the basis of a pressure-driven simulation, and the users' demand at the $i$-th node. $I_{s}$ can be calculated in both normal and critical operational scenarios.

\section{Case study and results}

The resilience metrics were applied to assess the hydraulic perturbation caused by different scenarios of Water Network Partitioning (WNP), obtained through the permanent closure of several pipes. The proposed metrics are tested for the real case study of the water distribution network (WDN) of Parete, a small town in the South of Italy with about 10,800 inhabitants. The WDN of Parete, reported in Figure 1, consists of 282 pipes, 182 nodes and 2 sources.

The following failure scenarios are evaluated: original network layout (no closed pipes) and nine partitioning layouts with a number of DMAs from 2 up to 10 and a number of closed pipes up to 20. The WNPs of Parete are obtained following two steps: step 1 - clustering, which provides the shape and dimension of clusters or DMAs; step 2 - dividing, which identifies pipes in which insert isolation valves and flow meters [15].

The clustering phase was performed by a Normalized Cut (NCut) algorithm [16]. NCut algorithm exploits the property of the Normalized Laplacian Matrix defined as $L_{r w}=D_{k}{ }^{-}$ ${ }^{1} L$, where the Laplacian matrix $L$ is the difference between the diagonal matrix $\mathrm{D}$, with the connectivity degrees of the nodes $(\mathrm{D}=\operatorname{diag}(\mathrm{K})$ and $\mathrm{K}$ is the degree of a node), and the adjacency matrix A (where elements $a_{i j}=a_{j i}=1$ indicate that there is a link between nodes $i$ and $j$ and $a_{i j}=a_{j i}=1$ otherwise).

The goal of NCut algorithm is to divide a network into $N_{d}$ clusters, in such a way as that the number of boundary edges or boundary pipes is minimized.

The dividing phase consists in a heuristic optimal positioning of flow meters and gate valves on pipes that connect different DMAs (defined as boundary pipes). This phase 
was accomplished by means of a Genetic Algorithm (GA) [17] to minimize the following constrained objective function $(\mathrm{OF})$ :

$$
\left\{\begin{array}{l}
F O: \min \left(n_{f m}\right) \\
\text { coinstraint: } I_{s}=100 \%
\end{array}\right.
$$

where, $n_{f m}$ is the number of flow meters, and $I s$ is the demand satisfaction rate (Eq. 6) assessed in the typical day of operation. Thus, the dividing phase guarantees fulfilment of users' total demand while inserting a low number of flow meters. The hydraulic simulations were carried out through the pressure driven approach (PDA) [12] during the typical day of operation with average demand at the nodes. Inside PDA, the important variables to set were $h_{\text {min }}$ and $h_{d e s}$, which are the minimum pressure heads for having an outflow to the users larger than 0 and for fully satisfying the users' demand at the generic node, respectively. The PDA of the network was carried out setting $h_{\min }$ and $h_{d e s}$ to $0 \mathrm{~m}$ and $19 \mathrm{~m}$, respectively in the formula proposed in [18]. In Table 1 , the number of flow meters $\left(n_{f m}\right)$ and gate valves $\left(n_{g v}\right)$ are reported for the studied failure scenarios.

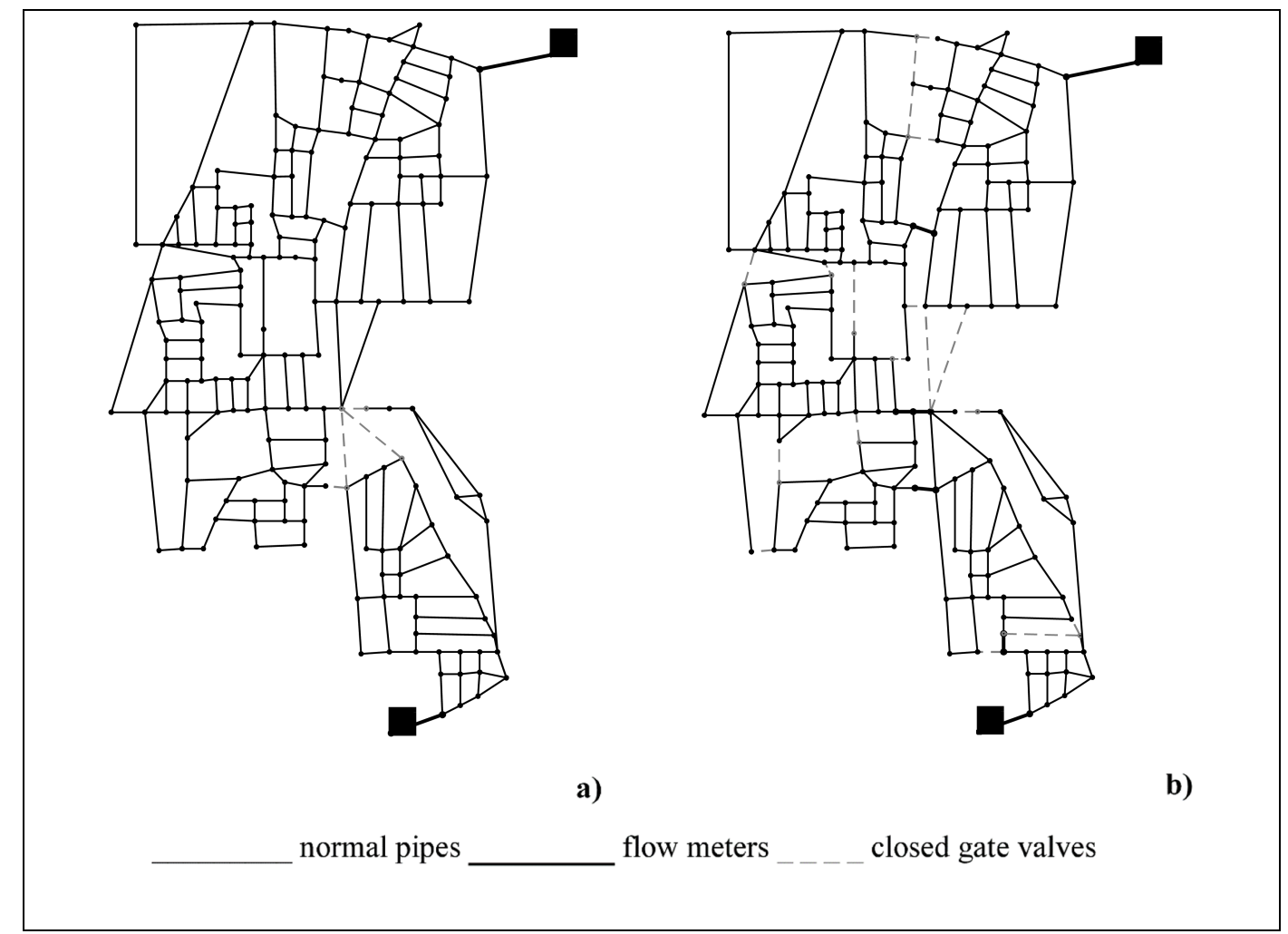

Figure 1 Partitioning layout of Parete water distribution network: a) with a number of DMAs $N_{d}=2$; b) with with a number of DMAs $N_{d}=6$. 
Table 1 Number of edge cut $\left(N_{e c}\right)$, number of flow meters $\left(n_{f m}\right)$ and number of gate valves $\left(n_{g v}\right)$ for each partitioning scenario

\begin{tabular}{lccc}
\hline$N_{d}$ & $N_{e c}$ & $n_{f i n}$ & $n_{g v}$ \\
\hline 2 & 4 & - & 4 \\
3 & 10 & 1 & 9 \\
4 & 16 & 2 & 14 \\
5 & 21 & 3 & 18 \\
6 & 23 & 5 & 18 \\
7 & 26 & 7 & 19 \\
8 & 29 & 8 & 21 \\
9 & 29 & 9 & 20 \\
10 & 31 & 11 & 20 \\
\hline
\end{tabular}

As showed in Table 1, the number of flow meters to place in network on boundary pipes is lower than or equal to the number of DMAs (except for $N_{d}=10$, where the former is slightly larger).

In Figure 2 and 3, the daily minimum, median and maximum values of energy efficiency index and global resilience failure are reported as a function of $N_{d}$ for the original network $\left(N_{d}=1\right)$ and all proposed partitioning layouts, computed during a typical operational day.

The minimum, $e_{m i n}$, and median, $e_{m e d}$, of energy efficiency index for partitioning scenarios are almost constant about equal to that of original network $\left(e_{\min }=0.31\right.$ and $e_{\text {med }}=0.61$ ), indeed it is not possible discern significant alterations. Proof of this is the horizontal trend of $e_{\min }$ and $e_{m e d}$ as shown in Figure 2. This is due to the effectiveness of the partitioning method, which is able to preserve well the energy delivery of the original layout. On the contrary, the maximum values, $e_{\max }$, corresponding to the peak hour of demand, are lower than the energy efficiency of original layout $\left(e_{\max }=0.80\right.$, $\left.N_{d}=1\right)$ while a minimum value occurs in case of $N_{d}=2\left(e_{\max }=0.76\right)$.

As for energy efficiency index, as reported in the Figure 3, the minimum of global resilience failure index, $G R F_{\min }$, are almost constant and equal to the original network $\left(G R F_{\min }=0.10\right)$. The median, $G R F_{\text {med }}$, shows a slight alteration of performance among the proposed failure scenarios although it is not strongly noticeable. Conversely, maximums of GRF $\left(G F R_{\max }\right)$ change considerably, indeed the index of original network is about $G F R_{\max }=0.50$ and the lower values is $G F R_{\max }=0.38$, that occurs for $N_{d}=2$.

The comparison between energy efficient index and global resilience failure index, reveals that GFR is more sensitive to the hydraulic alterations, caused by permanent partitioning, evidently the presence of term $h_{\text {des }}$ in Eq. 3 and 4, allows GFR to detect better the hydraulic behavior of network in case of large perturbations. 


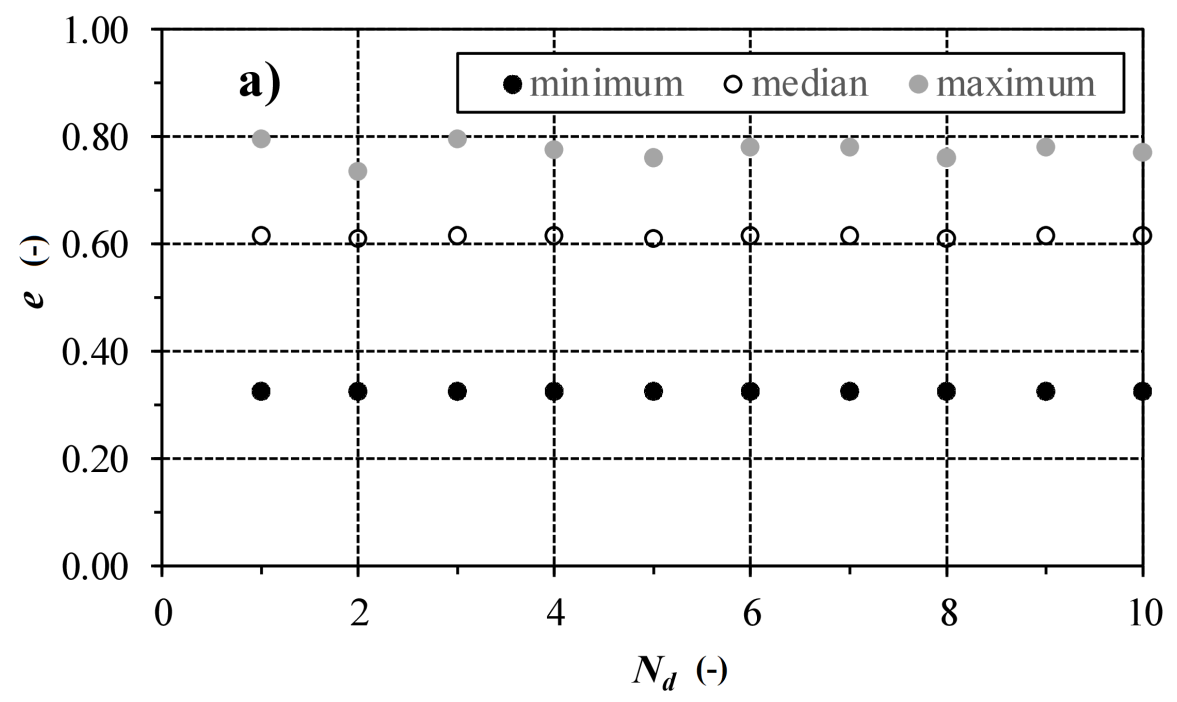

Figure 2 Minimum, median and maximum of energy efficient index

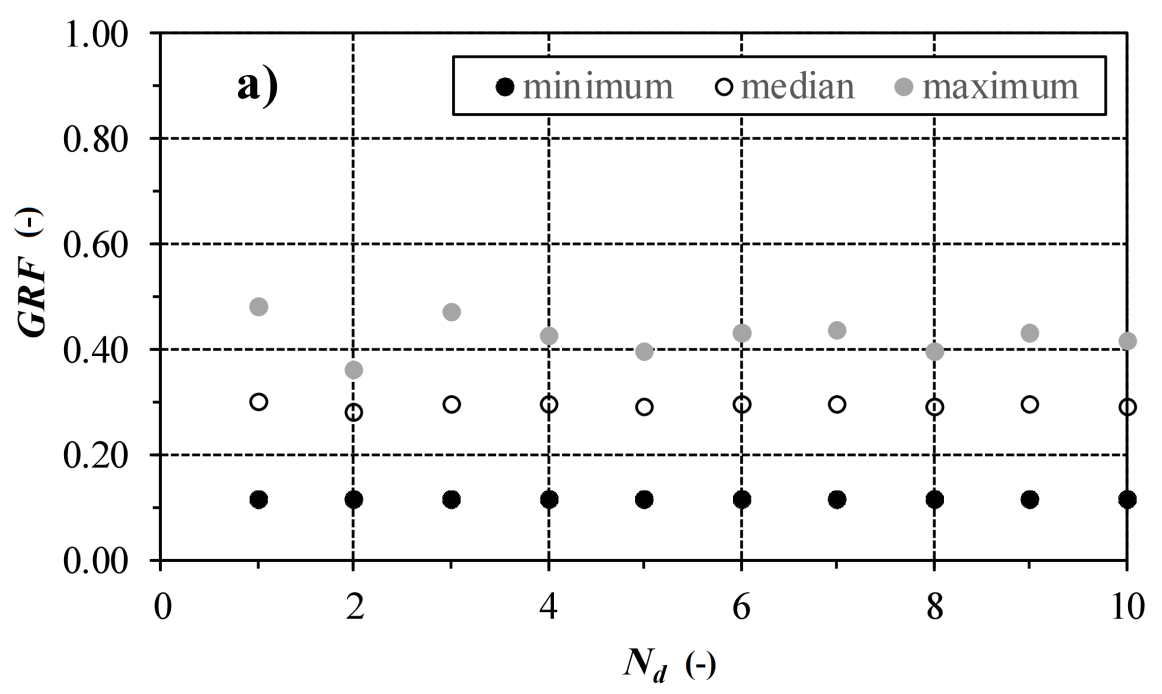

Figure 3 Minimum, median and maximum of $G R F$ index

In Figure 4 the loop diameter uniformity is reported; the values of $C_{u}$ are descending until $N_{d}=5$, but for a higher number of DMAs the trend became similar to that remarked for the maximum values of $e$ and $G R F$; thus the influence of $C_{u}$ could provide an insight into why some partitioning layouts, with lower number of closed pipe (e.g. $N_{d}=6$ and $N_{d}=8$ ), feature lower values of energy efficient index and $G R F$ than layouts with high number of closed pipes (e.g. $N_{d}=6$ and $N_{d}=8$ ). 


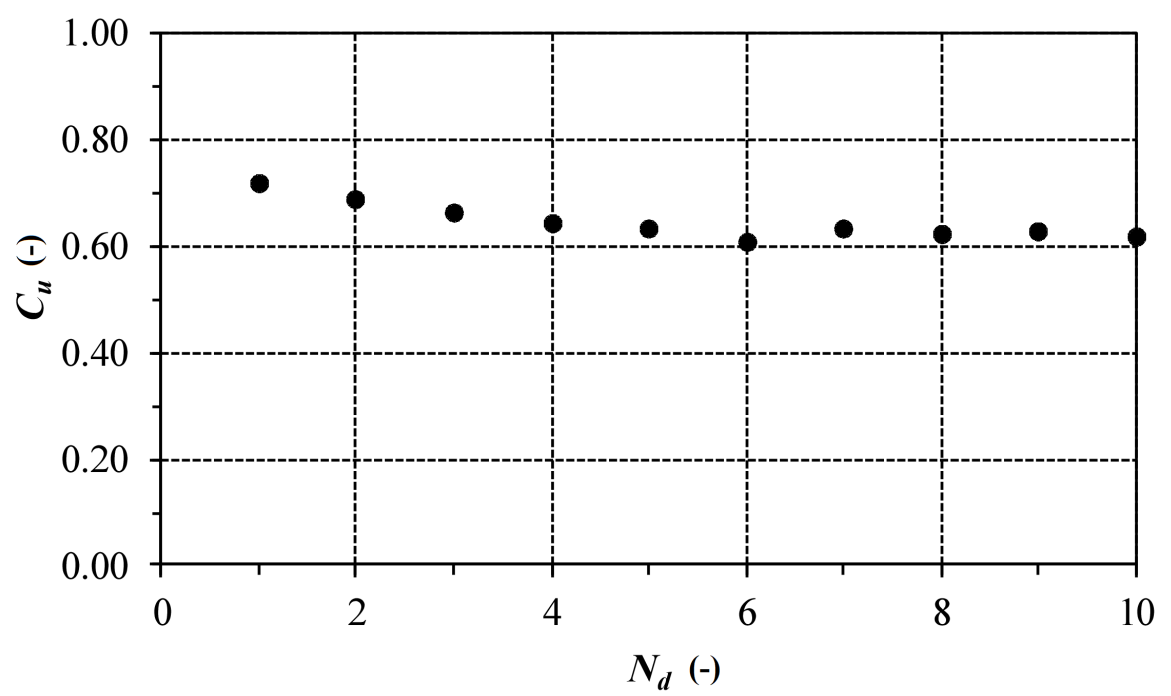

Figure 4 Trend of loop diameter uniformity

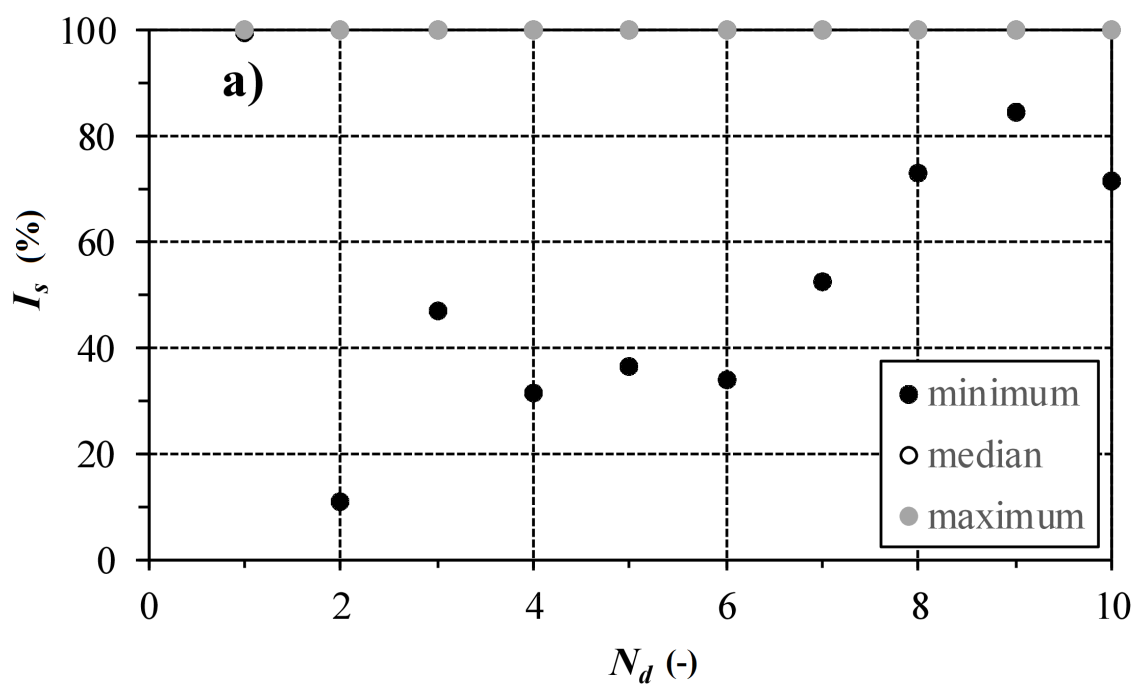

Figure 5 Minimum, median and maximum demand satisfaction rate

Figure 5 shows the trend of the demand satisfaction rate, computed in critical scenarios in which the WDN pipes are isolated one at a time. In these scenarios, the service disruption is assumed to occur for the whole duration of the day. In this figure, it is possible to remark that the minimum value of $I_{S}$ (represented by black dots in Figure 5 ) is always significantly lower than $100 \%$, apart from $N_{d}=1$ (unpartitioned network). This means that the demand is not fully satisfied when some pipes are isolated. An indepth analysis revealed that this is the case with the isolation of the feeding pipes 
connected to the network sources. The lowest value of $I_{s, \min }$ lies in correspondence to $N_{d}=2$, for which service disruption in either feeding pipe puts a large part of the WDN out of service. In fact, for $N_{d}=2$, the two districts are hydraulically independent (each one fed by a single source). This could be avoided if the water utility chose to open one boundary pipe in the middle of the WDN, while inserting an extra flow meter there. Interestingly, the compact resilience indices (see Figures 2 and 3), especially GRF assessed under peak demand conditions, were able to spot in advance the weaknesses of the partitioned layouts in terms of $I_{s, \min }$.

\section{Conclusions}

The paper presents a comparison between two different ways to compute the resilience of a partitioned water distribution network. The proposed indices were calculated for the partitioning layouts in order to define the most suitable tool able to provide a synthetic information about the behaviour of the entire network, in severe perturbation conditions, even with a high number of closed pipes, up to 20 for the WSN of Parete. Results show that the indices, especially the GRF assessed under peak demand conditions, is able to spot flaws in the network layouts obtained with partitioning.

\section{Reference}

[1] E. Todini, Looped water distribution networks design using a resilience index based heuristic approach Urban Water J 22000 115-122.

[2] T.T. Tanyimboh, Informational Entropy: a Failure Tolerance and Reliability Surrogate for Water Distribution Networks, Water. Resour. Manag. 31(10) 2017 3189-3204.

[3] A. Yazdani, P. Jeffrey, A complex network approach to robustness and vulnerability of spatially organized water distribution networks, Physics and Society, 15(2) (2010) 1-18.

[4] A. Di Nardo, M. Di Natale, C. Giudicianni, R. Greco, G.F. Santonastaso, Complex network and fractal theory for the assessment of water distribution network resilience to pipe failures, Wa Sci Technol, 17(4) 2017 ws2017124.

[5] W. Mays, Water distribution systems handbook McGraw-Hill, New York, 2000.

[6] D. N. Raad, A. N. Sinske, J. H. van Vuuren, Comparison of four reliability surrogate measures for water distribution systems design, Water Resour Res. 46(5) 2010 W05524.

[7] E. Creaco, M. Franchini, E. Todini, The combined use of resilience and loop diameter uniformity as a good indirect measure of network reliability, Urban Water J. 13(2) 2016 $167-181$.

[8] A. Gheisi, G. Naser, Water distribution system reliability under simultaneous multicomponent failure scenario, Am. Water Work Assoc., 106(7) 2014 E319-E327.

[9] A. Di Nardo, M. Di Natale, D. Musmarra, G.F. Santonastaso, V. Tzatchkov, V.H. AlcocerYamanaka, Dual-use value of network partitioning for water system management and protection from malicious contamination, J Hydroinform 201517 (3) 361-376.

[10] A. Di Nardo, M. Di Natale, C. Giudiciani, G.F. Santonastaso, V. Tzatchkov, J. M. Rodriguez Varela, Economic and Energy Criteria for District Meter Areas Design of Water Distribution Networks Software for partitioning and protecting a water supply network, Water 9(7) 2016463. 
[11] A. Gheisi, M. Forsyth, Gh. Naser, Water Distribution Systems Reliability: A Review of Research Literature, J. Water Res. Plan. Man. 142(11) 201604016047.

[12] E. Creaco, M. Franchini, E. Todini, Generalized Resilience and Failure Indices for Use with Pressure Driven Modeling and Leakage, J. Water Resour. Plann. Manage. 142(8) 2016 04016019.

[13] R. Dziedzic, B.W. Karney, Performance index for water distribution networks under multiple loading conditions, J. Water Resour. Plann. Manage. 142(1) 201604015040.

[14] E. Creaco, M. Franchini,. Fast network multi-objective design algorithm combined with an a posteriori procedure for reliability evaluation under various operational scenarios Urban Water J. 9 (6) 2012 385-399.

[15] A. Di Nardo, M. Di Natale, G.F. Santonastaso, S. Venticinque, An automated tool for smart water network partitioning, Water. Resour. Manag. 27(13) 2013 4493-4508.

[16] J. Shi, J. Malik, Normalized cuts and image segmentation, Ieee T. Pattern. Anal. 22(8) 2000 888-905.

[17] D.E. Goldberg, Genetic Algorithms in Search, Optimization and Machine Learning, Addison Wesley Boston, MA 1989.

[18] Ciaponi, C., Franchioli, L., Murari, E., Papiri, S. (2014). "Procedure for Defining a Pressure-Outflow Relationship Regarding Indoor Demands in Pressure-Driven Analysis of Water Distribution Networks." Water Resources Management, 29(3), 817-832. 